\title{
Foro Comunitario de Investigación. Una Herramienta Multipropósito
}

\section{Community Research Forum. A Multipurpose Tool}

\author{
Vicente Manzano-Arrondo \\ Universidad de Sevilla
}

\section{Resumen}

El foro comunitario es una herramienta ampliamante utilizada por organizaciones de la sociedad civil, en ocasiones junto con las administraciones públicas. Como instrumento de acción, el foro comunitario ha sido objeto de escasa atención académica o científica. A pesar de ello, es posible identificar algunas características comunes: (1) es un encuentro que tiene lugar en una comunidad de personas, (2) la comunidad participa en su organización y desarrollo, (3) el asunto que se aborda es relevante para la comunidad, y (4) hay al menos una fase de exposición, una fase de discusión, y una fase de conclusiones. Este trabajo presenta un tipo de encuentro, denominado Foro Comunitario de Investigación (FCI). Se trata de una aplicación de foro comunitario, donde el asunto que se aborda son los resultados de una investigación. Con este cometido, el trabajo define el $\mathrm{FCl}$, describe sus objetivos específicos, propone un esquema o método de realización, y ejemplifica su aplicación con un caso concreto.

Palabras clave: foro comunitario, foro comunitario de investigación, utilidad de la ciencia

\begin{abstract}
The community forum is a tool widely used by organizations of civil society, and sometimes with government. As a tool for action, the community forum has received little academic or scientific attention. However, it is possible to identify some common characteristics: (1) It is a meeting that takes place in a community of people; (2) the community is involved in its organization and development; (3) the topic addressed is relevant to the community, and (4) there is at least one phase of exhibition, a discussion phase, and a phase of conclusions. This paper presents a kind of meeting, called Community Research Forum (CRF). This is an application of community forum where the topic addressed is the result of research. With this function, the paper defines the CRF, describes its specific objectives, proposes a scheme or method for accomplishing it, and exemplifies its application to a particular case.
\end{abstract}

Keywords: community forum, community research forum, utility of science 


\section{Introducción}

Existen múltiples enfoques de investigación que cuentan con la participación ciudadana o de la comunidad como una de sus características fundamentales. Aunque el modelo más ampliamente conocido es la investigación-acción participativa (Brydon-Miller, Greenwood y Maguire, 2003), el panorama es más extenso. Se encuentran, entre otras posibilidades, las propuestas de investigación y acción colectiva (Botero, 2012), aprendizaje-servicio (Speck, 2001), unidades de acción comprometida (Manzano-Arrondo y Suárez, 2015), investigación militante (Conti, 2004), metodología comunicativa crítica (Gómez et al., 2008) o coproducción investigativa (Bialakowsky et al., 2007). Existen también ampliaciones participativas de métodos clásicos, como ocurre con las encuestas deliberativas (Fishkin, 1991), las encuestas participativas (Parrado, McQuiston y Flippen, 2005) o los cuestionarios de elección (van der Salm, van Knippenberg y Daamen, 1997).

Sin la motivación de transformar todo el proceso en una dinámica participativa, el mundo de la ciencia va reconociendo lenta y progresivamente los beneficios que se derivan de abrir un canal directo de comunicación y diálogo con la ciudadanía (Delgado, 2010) y nutrir su corpus con los saberes populares (Codina y Delgado, 2006). En esta línea, las propuestas de investigaciones participativas íntegras suponen una fuente de inspiración. Sin embargo, la complejidad de estas propuestas y la escasa familiaridad del mundo científico con ellas, son dos inconvenientes de peso que pueden estar justificando la lentitud con que van abriéndose camino en el ámbito del quehacer científico. Una posibilidad de solución es recuperar métodos, técnicas o dinámicas participativas específicas y divulgarlas en los medios académicos, acotadas y sistematizadas, de tal forma que se favorezca el contacto progresivo con estos procedimientos.

Este trabajo se inscribe plenamente en esa posibilidad de solución. Presenta una herramienta que, si bien nace de procesos íntegramente participativos, es susceptible de ser aplicada como etapa final en investigaciones convencionales. El documento propone la denominación de Foro Comunitario de Investigación $(\mathrm{FCl})$ para esa etapa final.

Con el objetivo de presentar el $\mathrm{FCl}$, el primer apartado delimita el concepto; el segundo, propone un método específico; y el tercero, ejemplifica esta herramienta en un caso aplicado que implica a los sectores primario (administraciones públicas), secundario (tejido empresarial) y terciario (movimientos y organizaciones ciudadanas), además de miembros del ámbito académico.

\section{Concepto y funciones}

La propuesta del $\mathrm{FCl}$ se inspira principalmente en las prácticas de foros comunitarios, que surgen del trabajo de comunidades por sí mismas o en combinación con otros estamentos. Cuenta, pues, con un origen muy aplicado, por lo que no se ha desarrollado con un interés académico. Esta circunstancia genera varias consecuencias. Entre ellas, las comunicaciones de los foros no tienen el hábito científico de acotar con precisión los conceptos, sino el interés resolutivo de utilizarlos según sea la situación. De este modo, el mismo término se refiere a un amplio abanico de posibilidades prácticas y, también, la misma práctica recibe denominaciones distintas. Otra consecuencia es que la vía mayoritaria de 
información y documentación no es la publicación en revistas científicas u otros espacios académicos, sino que se encuentra muy dispersa en forma de páginas web, octavillas, carteles, cursos y talleres.

La revisión de estos materiales muestra que se acude a la expresión foro comunitario con el ánimo de convocar a la ciudadanía en general, a un barrio o a una comunidad, para estimular la asistencia a un encuentro en el que se busca la cohesión y articulación ciudadanas, y se concreta la esperanza de acción colectiva en torno a un asunto concreto. Desde este enfoque común, se abre un abanico muy amplio de posibilidades. Cuando el foro comunitario es utilizado como herramienta de las administraciones públicas o de organizaciones sin ánimo de lucro de gran envergadura, se añade a lo anterior un estilo unidireccional: la administración u organización desea comunicar algo que afecta a la comunidad, exponiendo información y buscando la colaboración ciudadana para abordar el problema, en ocasiones con directrices muy concretas.

En lo que sigue, va a realizarse una síntesis ampliada a partir de los trabajos académicos de autores como Garrido, Luque-Ribelles y García-Ramírez (2013), Francisco y Shultz (2011) y Whitley (2002), así como de iniciativas de organizaciones como Education Fund (2015), Agency for Healthcare Research and Quality (2015), Forest Trends (2015), Global Health Advocacy Partnership (2015), Global Campaign (2015) y Voices (2014); fuentes que abordan expresamente la función y la organización de foros comunitarios. A la vez, autores como Montero (2009) o Fals Borda (1978), establecen criterios y fundamentos para organizar diálogos con las comunidades, que tienen una traducción fácil al contexto concreto de un $\mathrm{FCl}$. Estos documentos, a la vez que la experiencia personal del autor en la organización de este tipo de encuentros, son los que subyacen a la definición y estructuración del $\mathrm{FCl}$.

Un $\mathrm{FCl}$ es un encuentro que aborda el contenido, el significado y la relevancia de los resultados de una investigación, co-organizado al menos por el equipo del estudio y una comunidad, donde se busca facilitar el impacto social directo de la investigación, alimentar la aportación académica con el saber ciudadano, implicar a los presentes en vías de acción, promover tejido comunitario y estimular la coalición entre la academia y la comunidad para acciones futuras.

Esquemáticamente el $\mathrm{FCl}$ es una herramienta multipropósito que busca:

1. Devolver a la población los resultados de investigación obtenidos gracias a ella.

2. Aumentar la utilidad de la ciencia, estableciendo sinergias con la población general.

3. Mejorar la imagen de la ciencia y la academia a través de su percepción de utilidad e implicación con la ciudadanía.

4. Someter a juicio o evaluación los hallazgos de las investigaciones, mediante la reflexión y el debate con las personas que viven los problemas o los asuntos de investigación.

5. Alimentar las conclusiones del estudio con aportaciones de personas que pueden transmitir su saber práctico y su experiencia vivencial.

6. Estimular nuevas vías de investigación a partir de los retos que se acotan en ese espacio.

7. Aunar esfuerzos entre varios actores para garantizar la puesta en práctica de las directrices definidas tanto en la investigación como en el FCl. 
8. Resolver problemas de visibilidad y de concreción de actuaciones por parte de las administraciones públicas implicadas en las soluciones.

9. Facilitar el aprendizaje complejo de quienes participan en el proceso, mediante el contraste de sus perspectivas y de los resultados en torno a retos concretos y reales.

10. Construir comunidad y cohesión, no solo para las y los activistas que participan, sino también hacia los sectores a los que representan o de los que provienen.

11. Promover alianzas futuras, mediatas o inmediatas, orientadas a proyectos comunes concretos.

Por tanto, el $\mathrm{FCl}$ se inspira plenamente en la amplia experiencia de los foros comunitarios y en su impronta de acción, pero completa el mapa de motivaciones incluyendo aspectos relacionados con la investigación, con la puesta en práctica de los hallazgos y con las posibilidades de actuación conjunta en frentes futuros. Estas ventajas y posibilidades consecuentes no son exclusivas de un $\mathrm{FCl}$. Son compartidas con los enfoques que reúnen en un mismo entorno de trabajo y decisiones a la academia y a una comunidad o colectivo ciudadano (Anderson, 2003).

\section{Método}

La organización de un $\mathrm{FCl}$ implica un esfuerzo específico significativo. No puede ser improvisado y debería estar contemplado desde la fase de diseño de la investigación, pues consume recursos humanos y temporales, además de requerir en ocasiones un presupuesto específico. Si bien este documento tiene como uno de sus objetivos divulgar esta herramienta en el ámbito académico, pretende también cumplir con el interés de utilidad que caracteriza la dimensión de los foros comunitarios. Por este motivo, el método se expone con un nivel de detalle que facilita su concreción práctica. En consecuencia, el detalle aconseja recurrir a una exposición sintética, de tal forma que la extensión no llegue a ser excesiva.

En términos generales, se distinguen cuatro etapas, cada una de ellas, con sus requerimientos prácticos. Las dos primeras se inscriben en el proceso de preparación y diseño; la tercera, en la realización; la cuarta, en las actuaciones que se llevan a cabo una vez ha finalizado el encuentro.

\section{Antes 1: los preparativos}

1. Durante la fase de recogida de datos del estudio, se ha informado a los participantes (por ejemplo, personas entrevistadas) de que (1) se confeccionará un informe con los resultados y (2) se organizará un $\mathrm{FCl}$. Se les pregunta si desean recibir el informe por correo-e o noticias sobre cuándo y dónde tendrá lugar el $\mathrm{FCl}$. Se confecciona una base de datos con las direcciones electrónicas, cuya gestión está sujeta a la ley de protección de datos.

2. Una vez realizados los análisis de datos y a partir de los documentos amplios o exhaustivos que elabora el equipo de investigación, se confeccionan dos textos:

a) Un informe de divulgación. Contiene los resultados del estudio en un formato razonablemente breve, muy claro en su exposición y redacción, orientado a población general. 
b) Un resumen sintético. El informe de divulgación puede llegar a ser algo extenso y, por ello, se reduce la probabilidad de ser leído para un sector importante de la población. Por este motivo, en una página o poco más, se resumen los principales hallazgos, sin necesidad de acudir a cantidades concretas ni representaciones gráficas.

c) Tanto el informe de divulgación como el resumen sintético finalizan su redacción comunicando que va a tener lugar la convocatoria de un $\mathrm{FCl}$ y facilitando el modo en que las personas interesadas pueden informarse sobre ello.

3. Página web

En un espacio web específico (clásico, blog, Facebook, etc.), se organizan los siguientes elementos:

a) Una presentación que, a ser posible, no excede de un párrafo.

b) Enlaces a

- El documento de divulgación.

- El resumen sintético.

- Un cuestionario breve y abierto donde se solicitan básicamente dos informaciones: (1) impresiones derivadas de la lectura de cualquiera de los dos documentos anteriores y (2) sugerencias o predilecciones para organizar el FCl.

- Materiales: herramientas utilizadas en el estudio (por ejemplo, el cuestionario de la encuesta, el guión de las entrevistas o las hojas de observaciones), base de datos (que respeta la ley de protección de datos) y cualesquiera otros elementos que considere relevante el equipo de investigación.

c) Contacto con el equipo.

d) Banners o logos de las instituciones que han participado en el trabajo.

4. Diálogo con los co-organizadores

Se pretende organizar un encuentro de organización con todas las partes implicadas en la investigación o con interés en los hallazgos, para que de él surja el diseño del FCI. Si la reunión física con todas las partes no fuera viable, el equipo se entrevista con representantes de cada una de ellas

a) Las partes implicadas son, en su versión mínima, el equipo de investigación y una comunidad concreta. En su versión óptima, cuenta con representantes de la ciudadanía en general, de las administraciones públicas y de los sectores que puedan estar potencialmente interesados o implicados, como pueden ser comerciantes, medios de comunicación, instituciones sanitarias, asociaciones deportivas, religiosas, etc. según sea el asunto.

b) Antes del encuentro específico de organización o de las entrevistas cara a cara concertadas, se deriva hacia la web para facilitar la consulta del informe de divulgación y se orienta sobre el significado de un FCl.

c) Tras el encuentro específico o las entrevistas concertadas, hay que contar con respuestas o aportaciones de todas las partes respecto a:

- Modificación de los documentos de divulgación y resumen sintético, y del contenido de la web. 
- Impresiones sobre el contenido de la investigación y sus resultados.

- Directrices generales sobre el $\mathrm{FCl}$, en términos de qué hay que buscar y qué hay que evitar.

- Preferencias respecto al contenido del FCl: guión, formato de trabajo, materiales, etc.

- Plazos a cumplir para las fases restantes.

- Qué papel va a tener su organización o institución en el FCl.

- Cómo va su organización o institución a divulgar el FCl.

d) Con esta información se elabora un informe sintético (documento de diseño) que es compartido con las partes y, en su caso, modificado tras su lectura, con el objetivo de que los co-organizadores cuenten con información completa relevante. Este informe contiene la identidad de las personas de cada organización implicada, que van a participar en el diseño concreto del FCl.

5. Divulgación. La web es publicitada durante el tiempo acordado en el punto anterior. La divulgación busca ir creando un clima de interés por los resultados de la investigación y por la posibilidad de asistir al $\mathrm{FCl}$.

a) Utilización de los canales propios de cada co-organizador y de colaboradores (organizaciones e instituciones que no forman parte del diseño del FCl pero que aportan sus posibilidades de comunicación)

b) Enlaces compartidos con webs amigas.

c) Anuncio en blogs y páginas Facebook de la ciudad o comunidad.

d) Recurso de los medios de comunicación locales convencionales.

6. Reuniones de organización. Las personas concretas que han adquirido el compromiso de coorganizar el $\mathrm{FCl}$ se reúnen conjuntamente o por partes, en una o varias ocasiones, hasta traducir las directrices del documento en un diseño concreto y detallado.

\section{Antes 2: el diseño}

7. Redacción precisa de los objetivos del $\mathrm{FCl}$, distinguiendo los principales de los secundarios.

8. Secuenciación precisa, que incluye qué, quién y cómo en:

a) Bienvenida a los asistentes y presentación de la sesión, donde se comunican los objetivos, las partes que lo organizan, las expectativas de la organización y la dinámica que se va a seguir hasta el último de los actos del encuentro.

El acto de bienvenida incluye unos consejos actitudinales. Así, por ejemplo, es frecuente que muchas personas tiendan a utilizar el encuentro como un espacio donde desahogar su situación particular, o culpar a otras partes, sin ningún ánimo constructivo. Si bien esta circunstancia es humanamente comprensible, deriva fácilmente en la inutilización del $\mathrm{FCl}$. Este y otros problemas pueden ser anticipados, solicitando a las y los presentes que se concentren en una actitud propositiva. Una posibilidad de prevención es acudir a un folleto muy breve y atractivo, con un decálogo o listado de consejos para afrontar la participación en el $\mathrm{FCl}$.

b) Resolución de dudas sobre los objetivos y dinámica del acto. 
c) Exposición de los resultados de investigación

Es conveniente que exista un soporte adecuado para exponer los resultados, principalmente representaciones gráficas y algunas tablas. Quien expone esta información ha de cuidar de suministrar una imagen de conjunto, en todo momento, conectando unos resultados con otros.

d) Desarrollo

Cada $\mathrm{FCl}$ va a adoptar una forma precisa diferente. Lo que sigue se expone con el ánimo de comunicar un proceso generalista que suele funcionar bien en la práctica, pero que deberá ser modificado e incluso sustituido en su totalidad, según sea el asunto del FCl y las características propias de la comunidad con la que se trabaja.

- Fase 0 o previa: reflexión libre.

Tras la exposición de los resultados conviene organizar un tiempo libre, quizá con algún tentempié. Las y los participantes se mueven por la sala y conversan libremente sobre lo escuchado. La sala puede contener en sus paredes algunos carteles con los principales hallazgos, de tal forma que las reflexiones cuentan con un soporte.

- Fase 1: concreción de las situaciones de partida y llegada.

Se forman grupos relativamente pequeños de discusión, entre 5-9 personas, a la que se suma un miembro de la organización que modera, toma nota y aclara dudas. Cuanto más diversos sean estos grupos, mejor. Antes de comenzar, puede establecerse alguna estrategia de identificación (tarjetas de colores, por ejemplo), que asocia a cada persona con un grupo de trabajo. El cometido del grupo es definir una situación de partida, es decir, un diagnóstico del asunto a partir de los resultados de la investigación y de sus propias experiencias. Acto seguido, hay que definir cuál es la situación a la que les gustaría llegar, o situación de llegada. Esta segunda configuración procura ser realista, en el sentido de que el grupo considera que se cuenta con posibilidades para hacerla realidad.

- Fase 2: plenario de tránsitos partida-llegada. Se ponen en común las situaciones de partida y llegada, con las matizaciones que se deseen, hasta llegar al dibujo de convergencias e incluso de divergencias sobre ambos puntos, que el plenario establece como dibujo común.

- Fase 3: barreras, soluciones y agentes. Se repiten los grupos de trabajo (según el tiempo disponible, se forman de nuevo, o se mantienen los anteriores). Con las conclusiones del plenario, el grupo identifica barreras o problemas que pueden encontrarse en la práctica del tránsito entre la partida y la llegada. Del mismo modo, cada barrera se asocia con una o varias estrategias de solución. Por último, se identifican los agentes sociales que han de participar en las soluciones.

- Fase 4: plenario de conclusiones. Se ponen en común las aportaciones de los diferentes grupos, identificando las coincidencias y resolviendo o considerando expresamente las divergencias.

e) Evaluación y despedida.

Se solicita a las y los participantes que respondan a un cuestionario de impresiones sobre el foro, que incluye también la posibilidad de dejar el correo electrónico u otra forma de contacto que permita informar sobre las acciones, solicitar colaboraciones, etc.

9. Presupuesto y espónsor

El FCl va a implicar con seguridad algún coste. Es posible que la forma de organizarlo permita 
que los costes se asuman en especie por parte de las entidades que participan en la organización, como ocurre si alguna pone el local, otra realiza fotocopias, etc. No obstante, este aspecto debe ser expresamente considerado, puesto que es posible que se requiera algún aporte monetario y que, por tanto, haya que definir quién lo aporta y en qué condiciones.

10. Cuando el FCl se desarrolla a lo largo de varias horas, aparece la necesidad de ofrecer comida y bebida, circunstancia que obliga a precisar cómo se concreta su compra y disposición en el acto. La situación óptima es que el presupuesto de investigación contemple los gastos derivados de una fase final con $\mathrm{FCl}$, de tal forma que es la entidad que financia el estudio la que aporta también la seguridad monetaria para la realización correcta del encuentro. Debido al ánimo del $\mathrm{FCl}$, no debería organizarse mediante gastos superfluos. Aborda la definición y solución de algún asunto de interés social que sistemáticamente se desarrolla en condiciones de desigualdad. Los gastos superfluos pueden ser observados como una agresión o un desequilibrio evidente entre el equipo de investigación o los co-organizadores, por una parte, y la comunidad, por otra.

\section{Registro de participantes}

Es recomendable solicitar que las personas que deseen asistir al $\mathrm{FCl}$ se registren previamente, lo que puede concretarse a través de un formulario en la web o mediante contacto por correoe o teléfono. En este caso, lo relevante no es la identidad sino el número. Por este motivo, no se interroga profusamente a esta persona, sino que se le pide una información mínima, suficiente como para contar finalmente con una estimación de asistentes. Ello permitirá adecuar los servicios, el local, el diseño de grupos de trabajo, la comida, etc.

\section{Mientras: el encuentro}

12. Antes del acto:

a) Alguien de la organización ha de llegar al lugar del encuentro con tiempo suficiente como para comprobar que todo está en orden, funciona el equipo de sonido, las mesas y sillas están dispuestas como corresponde a las dinámicas de grupos de trabajo, etc.

b) Es posible que haya que recoger a ponentes, o a participantes con movilidad reducida, entre otras posibilidades, circunstancia que ha tenido que contemplar el equipo organizador.

13. Durante el acto:

a) El equipo de organización cuenta con distintivos que permiten a los participantes acudir a sus miembros para resolver dudas, orientarse o realizar comentarios o sugerencias sobre el desarrollo del acto.

b) De forma óptima, el equipo cuenta con una persona en cada grupo de trabajo y al menos otra que está supervisando todo el proceso.

c) El miembro del equipo en cada grupo de trabajo procura:

- Recordar los cometidos del grupo, ya mencionados en la presentación del acto.

- Guiar una ronda inicial de presentaciones.

- Iniciar el trabajo o el asunto, que comienza acordando la dinámica que prefiere el grupo para trabajar (por ejemplo, acotando tramos de tiempo para el desarrollo, abordando subtemas de manera secuencial, o en paralelo mediante una tabla específica, etc.). 
- Moderar, procurando (1) frenar las intervenciones prolongadas, (2) estimular la participación, (3) corregir el rumbo cuando se desvía del cometido, (4) cuidar la dinámica que el grupo ha acordado en su primera decisión, (5) sintetizar los puntos a los que se va llegando, (6) advertir cuando se está entrando en una discusión cíclica, y (7) en definitiva, garantizar el establecimiento de conclusiones.

d) En los plenarios, el equipo de organización adapta el guión a la situación real, decidiendo los tramos de tiempo de las dinámicas, como ocurre con el tiempo disponible a cada moderador de grupo para exponer las conclusiones.

14. Antes de la despedida, el miembro del equipo que está a cargo de este punto, ha conversado con los moderadores de grupo, recogiendo sus impresiones sobre el desarrollo del acto, para mencionar estos aspectos en su breve discurso.

15. Despedida, que contiene, además de referencias al punto anterior:

a) Las impresiones del equipo organizador.

b) Un repaso rápido por el desarrollo y las conclusiones.

c) Una felicitación por el trabajo realizado.

d) El anuncio de los siguientes pasos que, sin ánimo de comprometer a los asistentes, están abiertos para la participación de quienes así lo deseen.

e) Las vías de comunicación que se van a utilizar para informar sobre el desarrollo de las decisiones adoptadas en el $\mathrm{FCl}$.

f) La solicitud de que los participantes, antes de abandonar el espacio del encuentro, respondan a un cuestionario breve, volcando sus impresiones.

\section{Después: tras el acto}

16. Evaluación

Las impresiones del equipo, las declaraciones literales recogidas por quienes moderaron los grupos, y las respuestas al cuestionario final, se estudian para dar forma a un documento específico de evaluación sobre el acto.

17. Reunión

El equipo de organización se reúne pasado un tiempo prudencial tras el acto, acordado de antemano, para:

a) Interpretar los resultados de evaluación.

b) Establecer conclusiones propias sobre el FCl.

c) Acotar o redefinir las responsabilidades o compromisos adquiridos por cada parte, asociando en cada punto algún criterio concreto como son los plazos de tiempo o la secuenciación de logros.

18. Informe

Con toda la información anterior, el equipo de investigación elabora un informe específico del $\mathrm{FCl}$, que es enviado al equipo organizador y que se cuelga en la página web del $\mathrm{FCl}$ como material final o de cierre. El informe, no obstante, contiene el listado de compromisos adquiridos, asociados con las partes responsables de ello.

19. Seguimiento

El seguimiento de los acuerdos no es responsabilidad expresa del equipo de investigación, 
sino de las partes co-organizadoras, que incluyen a este equipo, como también a la comunidad y a otros agentes participantes, como pueden ser las administraciones públicas. El seguimiento de los compromisos, si bien es un cometido fundamental para garantizar la utilidad del $\mathrm{FCl}$ y para no frustrar expectativas legítimas asociadas a ello, excede las competencias expresas del $\mathrm{FCl}$.

\section{Una Aplicación en El Puerto de Santa María}

El Puerto de Santa María es una ciudad con cerca de 90 mil habitantes ${ }^{1}$, situada en la bahía de Cádiz (sur de España), con un centro histórico fundado en el siglo XIII y que toma forma entre los siglos XIII y XVIII (Barros, 2001), con motivo de la actividad que se genera en torno a la desembocadura del río Guadalete (López y Ruiz, 2003). Con posterioridad, muy especialmente desde mediados del siglo XX, la ciudad ha crecido, diseminándose en algunas zonas delimitadas: (1) residenciales costeras, (2) diseminadas de interior y (3) barriadas de densidad similar al centro histórico, de calidades muy dispersas, y situadas especialmente hacia el Norte.

A través de un convenido suscrito entre la Universidad de Sevilla y la Asociación de Empresarios Centro Comercial Abierto, se puso en marcha una encuesta a población general ( $n=350$, con muestreo polietápico de cuotas de edad y sexo en última etapa) en la ciudad de El Puerto de Santa María, durante la primavera de 2015. En la encuesta se recabó información sobre cómo la ciudadanía ve su ciudad, qué se podría hacer para solucionar sus problemas y otros asuntos relacionados con ideología (concepciones de ciudadanía y encuadres ideológicos) y consumo (movilidad, espacios y estilos de consumo, huella ecológica, etc.). La encuesta se complementó con entrevistas a representantes de 25 organizaciones de la sociedad civil sobre motivos similares.

Uno de los resultados de la encuesta fue un alto nivel en los factores de negatividad (visión negativa sobre la ciudad) y fatalismo (sensación de que no puede hacerse nada). A raíz de ello, diversos contactos con representantes del gobierno local y de organizaciones de la sociedad, confirmaron las impresiones iniciales de que se trataba de un estudio con interés general y que podría ser utilizado como base para realizar un diálogo con la ciudadanía.

Se siguió con razonable literalidad el esquema descrito en el apartado del método, si bien se encontraron algunos problemas o barreras en el desarrollo. Así, no fue posible reunir al gobierno local, movimientos sociales y tejido empresarial juntos en un encuentro de co-organización del $\mathrm{FCl}$, por lo que el punto 4 del método tuvo que desarrollarse mediante entrevistas específicas para cada parte. Estas entrevistas confirmaron el interés en el estudio y la pertinencia del $\mathrm{FCl}$, aunque mostraron algunas reticencias. Estas provenían especialmente del tercer sector, es decir, de algunos representantes de organizaciones de la sociedad civil, que consideraban un trabajo infructuoso dialogar con el gobierno local o bien una actividad incómoda la relación con el tejido empresarial. No

1 Cifra oficial de 88.700, según la revisión del padrón municipal del Instituto Nacional de Estadística, a 1 de enero de 2014: http://www.ine.es/jaxiT3/Datos.htm?t=2864 
obstante, al profundizar en las rondas de conversaciones y observar el interés común por mejorar la situación de la ciudad, estas resistencias fueron venciéndose.

Tras un mes de conversaciones, se consiguió constituir un grupo de organización formado por representantes del equipo de investigación, del gobierno local, de la asociación de comerciantes y de movimientos sociales. El equipo trabajó mediante un documento web compartido en abierto, y una única reunión presencial, donde se terminaron de concretar todas las decisiones relevantes para la organización del $\mathrm{FCl}$. Este evento tuvo lugar un mes después de la reunión física. Fue publicitado por las redes sociales, la prensa local ${ }^{2}$ y con el asesoramiento del gabinete de prensa del Ayuntamiento. A su vez, se confeccionó una página web $^{3}$ conteniendo la información a que se refiere el punto 3 del método y en el que se anunciaba el Foro, así como el enlace al registro de asistentes.

El $\mathrm{FCl}$ contó con la participación de 15 representantes de asociaciones diversas de la ciudad, organizado a lo largo de cuatro horas, siguiendo el esquema:

1. Bloque de presentación

a) Bienvenida a la jornada

b) Toma la palabra un representante de cada sector (gobierno local, asociación de comerciantes y organización de la sociedad civil)

C) Se realiza una descripción del estudio, especialmente de los resultados.

2. Bloque 1 de trabajo. Se forman grupos de discusión y debate que, a raíz de la presentación y de su propia experiencia e informaciones varias, acotan respuestas para dos preguntas: ¿Qué está pasando en El Puerto? ¿Por qué?

3. Bloque 2 de trabajo. Los moderadores de los grupos realizan una puesta en común para las conclusiones del primer bloque y vuelven a los grupos para comunicarlo. Se inicia otra fase inicial, esta vez para responder a la pregunta ¿Qué hacer para solucionar estos problemas?

4. Puesta final en común.

A partir de las aportaciones en los bloques de trabajo, se elaboró un informe específico de siete páginas, en donde se articulaban las conclusiones en torno a las preguntas de diagnóstico y a las soluciones. El informe estuvo en estudio en las organizaciones participantes durante dos semanas, tras cuyo transcurso se contó con diversas matizaciones, sugerencias y añadidos que enriquecieron la segunda versión de texto. Este documento final, fue divulgado por los mismos medios que se

utilizaron para la investigación original y para el foro. Es importante destacar que las diferentes

2 Radio Puerto: http://www.radiopuerto.fm/noticias/2015/11/19112015\%20-\%20la-jornada-el-puertoen-nuestras-manos-abrira-el-debate-para-mejorar-la // Andalucía Información:

http://andaluciainformacion.es/el-puerto/550029/el-puerto-visto-desde-su-gente/ // Diario de Cádiz: http://www.diariodecadiz.es/article/elpuerto/2158349/estudio/acusa/los/portuenses/negativismo/fat alista/se/sometera/debate.html y http://www.diariodecadiz.es/article/provincia/2159870/los/valientes/centro.html // El Puerto Actualidad: http://elpuertoactualidad.es/?p=140691\&cpage $=1$

3 http://personal.us.es/vmanzano/elpuerto/ 
organizaciones adquirieron compromisos concretos respecto a soluciones también concretas, de tal forma que en el momento de redactar estas líneas, tales soluciones se están llevando a cabo.

\section{Discusión y Conclusiones}

A lo largo de este trabajo, se ha justificado y sistematizado el $\mathrm{FCl}$ como un proceso que va más allá de ser únicamente un instrumento científico o de investigación. Se trata de una herramienta con varios propósitos que afecta a varios ámbitos: (1) abunda en la tendencia actual de abrir el diálogo entre ciencia y ciudadanía; (2) cuenta con importantes beneficios para la dimensión de la ciencia; (3) se instala bien en la motivación de utilidad pública; (4) ayuda a construir implicación ciudadana mediante la participación en asuntos concretos; y (5) genera conclusiones útiles para la toma de decisiones en las administraciones públicas y las organizaciones implicadas. Abre, por tanto, el abanico de posibilidades de una acción de investigación, en la línea que Fine (2006) establece para su propuesta de generalizabilidad provocativa: mover los resultados de investigación hacia fuera de los muros del contexto investigador, tanteando los efectos que ello provoca.

En lo que se refiere a los beneficios para el mundo científico, el $\mathrm{FCl}$ implica la posibilidad de mejorar algunos aspectos pertinentes a la calidad y relevancia de las investigaciones. Así,

1. Implica un incremento de la validez, en línea con la conceptuación del rigor en investigaciones cualitativas, que define Tracy (2010):

a) Las conclusiones no solo se someten a un posible debate (que no siempre es real) con la comunidad científica, sino que el público que juzga se amplia sensiblemente.

b) La ciudadanía contrasta los hallazgos de investigación con su realidad cotidiana, sometiendo las afirmaciones a un fuerte control de validez de criterio.

2. Mejora la imagen pública de la ciencia, al comunicar la utilidad práctica de sus resultados de investigación.

3. Estimula nuevas líneas de trabajo, ya que la discusión abierta permite que afloren problemas, incoherencias o retos que difícilmente serían observables dentro de las fronteras exclusivas de la ciencia.

4. Favorece la transdisciplinariedad...

a) ....al enfrentarse a problemas reales que no entienden de disciplinas $y$

b) ...al requerir conocimientos de origen diverso para garantizar que no solo la investigación en sí misma, sino el proceso de diálogo y la articulación operativa de conclusiones y compromisos, puedan contar también con el respaldo del conocimiento científico.

Las ventajas de un $\mathrm{FCl}$ no solo se inscriben en el contexto académico. Resuelve, por ejemplo, algunas críticas volcadas sobre la investigación-acción participativa, acusada de generar únicamente conocimiento político, pero no científico (Balcazar, 2003).

Por el lado negativo o de los inconvenientes, cabría mencionar principalmente: el consumo de recursos, la utilización sesgada del instrumento y las resistencias comunitarias. Respecto a primer inconveniente, la inclusión de una fase $\mathrm{FCl}$ en cualquier investigación, implica un esfuerzo mayor, más 
conocimientos, más implicación temporal $y$, en algunos casos, más presupuesto. Estos inconvenientes, especialmente el último, pueden contar con dificultades de solución en el sistema académico, considerando que es poco o nada propicio para sumergirse en procesos participativos comunitarios (Greenwood, 2012; Thiollent, 2011). En el ánimo de este documento se encuentra disminuir los inconvenientes a través de la sistematización y ejemplificación del proceso, y visibilizando su viabilidad práctica. Existe además, el convencimiento derivado de la experiencia, de que una vez que las personas de ciencia se implican en prácticas de $\mathrm{FCl}$ o instrumentos similares que suponen un contacto directo con las comunidades, no solo reducen las resistencias debidas al desconocimiento, sino que se genera con facilidad una especie de encantamiento que aumenta las probabilidades de repetir la experiencia.

Respecto al sesgo en la utilización, el FCl podría ser interpretado como una estrategia para mantener las relaciones de desequilibrio de poder entre ciencia y ciudadanía, al hilo del enfoque crítico y reflexivo que describe Delgado (2010). Según este enfoque, tales estrategias buscan recuperar la confianza de la ciudadanía para mantener el estatus de privilegio de la ciencia. En la misma línea se encuentran los textos que advierten de la tendencia de la academia a imponer su liderazgo y saberhacer en los procesos de aparente relación dialógica que establece con la parte comunitaria (Smith et al. 2010; Strier, 2011), pues está habituada a manejarse en el par experto-ignorante (Botero, 2012). Arieli, Friedman y Agbaria (2009) extienden esta crítica al personal técnico en general, cuyas buenas intenciones provocan, no pocas veces, más perjuicio que beneficio en los procesos de construcción de cohesión y participación comunitarias. Este trabajo no puede discutir estas críticas, que cuentan con un fuerte soporte empírico. El FCl podría ser utilizado con éxito bajo ese enfoque de desequilibrio de poder. No obstante, no es la intención de su diseño y divulgación. El lugar natural del $\mathrm{FCl}$ es inserto en un proceso participativo (Garrido, Luque-Ribelles y García-Ramírez, 2013) que incluye la relación con la ciudadanía desde el primer momento, es decir, desde la definición de los objetivos del estudio (estadio de alto compromiso en investigación, según el esquema de Manzano-Arrondo, 2012) y que culmina en un proceso que Mori (2008) denomina de diseminación, en el que se debate con la comunidad los resultados y los siguientes pasos a realizar. No obstante, ni siquiera los modelos participativos clásicos, como la investigación-acción ya mencionada, cuentan con una visibilidad apreciable en el mundo de las ciencias. En ese panorama, no solo $\mathrm{FCl}$ constituye un movimiento reformista (es decir, no revolucionario) para matizar las relaciones de poder ciencia-ciudadanía, sino que puede ser utilizado como palanca de cambio para entrar en una transformación más profunda, una vez que ambos actores han comenzado a trabajar juntos $y$, por tanto, han roto la extensa barrera del desconocimiento mutuo.

Las resistencias de la ciudadanía en general y las comunidades y organizaciones de la sociedad civil en particular, para establecer procesos participativos con la academia, quedan justificadas por las experiencias prácticas. Existe una desconfianza históricamente construida en torno al comportamiento científico, poco interesado en los problemas reales de las personas reales en contextos reales (Conti, 2004; Malo, 2004; Manzano-Arrondo, 2012). Buena parte de los contactos entre ambas dimensiones no son positivos, pues la ciencia dispone y las comunidades, cuando son llamadas, fácilmente no encuentran incentivos para la participación (Espinosa, 2001) ni para que su 
saber sea considerado (Codina y Delgado, 2006). Las resistencias se vencen cuando se consigue realizar prácticas exitosas, al menos en su dinámica; es decir, cuando la parte comunitaria vive la experiencia de un trabajo realmente equilibrado (Montero, 2009) y dialógico (Gómez et al., 2006). La posibilidad de que el $\mathrm{FCl}$ sea utilizado para reforzar desequilibrios de poder no puede ser prevista desde el diseño ni la realización del foro. La única garantía es la existencia de vocación dialógica, sincera y abierta por parte, principalmente, del equipo de investigación. Ninguna técnica puede sustituir la actitud ética.

En definitiva, pues, el $\mathrm{FCl}$ permite la oportunidad a todas las partes integrantes de lograr objetivos concretos relacionados específicamente con sus respectivos cometidos generales, resultado que requiere de un cuidadoso proceso previo de trabajo, de un desarrollo coherente y de una relación posterior que cumpla con los compromisos adquiridos.

\section{Información del autor/Author Information:}

Comunicación relativa a este artículo debe estar encaminada a Vicente Manzano-Arrondo, Universidad de Sevilla. Email: vmanzano@us.es

\section{Referencias}

Agency for Healthcare Research and Quality (2015). The AHRQ Community Forum Deliberative. Recuperado de http://www.effectivehealthcare.ahrq.gov/ehc/assets/File/DemonstrationMethods-Deliberative-130213.pdf

Anderson, S. (2003). Engaging students in community-based research: A model for teaching social work research. Journal of Community Practice, 10, 71-87. DOI: http://dx.doi.org/10.1300/J125v10n02_05

Arieli, D., Friedman, V.J. \& Agbaria, K. (2009). The paradox of participation in action research. Action Research, 7(3), 263-290. DOI: http://dx.doi.org/10.1177/1476750309336718

Balcazar, F.E. (2003). Investigación acción participativa (iap): Aspectos conceptuales y dificultades de implementación. Fundamentos en Humanidades, 4, 59-77.

Barros, J.R. (2001). El Puerto de Santa María. La ciudad renovada. Cádiz: Grupo Publicaciones del Sur.

Bialakowsky, A., Costa, M.I., Patrouilleau, M., Martínez, R.S. \& López, A.I. (2007). Capitalismo y método. Alternativas de la coproducción investigativa. Revista Laboratorio-on line. Estudios sobre Cambio Estructural y Desigualdad Social, 8(19).

Botero, P. (2012). Investigación y acción colectiva -IAC- Una experiencia de investigación militante. Utopía y Praxis Latinoamericana, 17(57), 31-47. 
Brydon-Miller, M., Greenwood, D. \& Maguire, P. (2003). Why action research?. Action Research, 1(1), 928. DOI: http://dx.doi.org/10.1177/14767503030011002

Codina, S. \& Delgado, C.J. (2006). La revolución contemporánea del saber y la complejidad social. Buenos Aires: Consejo Latinoamericano de Ciencias Sociales.

Conti, A. (2004). La encuesta como método político. En M. Malo (Eds.), Nociones Comunes (pp. 55-65). Madrid: Traficantes de Sueños.

Cuesta, M., Font, J., Ganuza, E., Gómez, B. \& Pasadas, S. (2008). Encuesta deliberativa. Madrid: Centro de Investigaciones Sociológicas.

Delgado, A. (2010). ¿Democratizar la ciencia? Diálogo, reflexividad y apertura. Revista lberoamericana de Ciencia, Tecnología y Sociedad, 5(15), 9-25.

Education Fund (2015). Opportunity through affirmative action. Planning a Community Forum on Affirmative Action. Recuperado de http://www.civilrights.org/equalopportunity/action/fair_chance_community_forum.pdf

Espinosa, H. (2001). Incremento de la capacidad comunitaria y del empoderamiento de las comunidades para promover la salud. Revista de la Facultad Nacional de Salud Pública, 19(1), 41-56.

Fals Borda, O. (1978). El problema de cómo investigar la realidad para transformarla. Recuperado de http://www.ts.ucr.ac.cr/binarios/pela/p1-000411.pdf

Fine, M. (2006). Bearing witness: Methods for researching oppression and resistance - A textbook for critical research. Social Justice Research, 19(1), 83-108.

Fishkin, J. (1991). Democracy and deliberation: new directions for democratic reform. New Haven: Yale University Press.

Forest Trends (2015). Foro Comunitario para el Intercambio y Monitoreo sobre REDD+ y PSA. Recuperado de http://forest-trends.org/documents/files/doc_3043.pdf

Francisco, V. \& Shultz, J. (2011). Community Tool Box. Llevar a cabo foros públicos y sesiones de escucha. Recuperado de http://ctb.ku.edu/es/tabla-de-contenidos/valoracion/valorar-lasnecesidades-y-recursos-comunitarios/foros-de-conducta-publica/principal

Garrido, R., Luque-Ribelles, V. \& García-Ramírez, M. (2013). La investigación-acción participativa como estrategia de intervención psicosocial. En J. Buades (Eds.), Manual de intervención comunitaria en barrios (pp. 102-123). Valencia: Fundación CeiMigra.

Global Campaign (2015). Cómo organizar un foro comunitario sobre microbicidas. Diez pasos. Recuperado de http://www.global-campaign.org/clientfiles/Inst8-ForoCom\%5BE\%5D.pdf

Global Health Advocacy Partnership (2015). Organize a Community Forum. Recuperado de http://www.action.org/documents/How_to_Organize_a_Community_Forum.pdf

Gomez, J., Latorre, A., Sánchez, M. \& Flecha, R. (2006). Metodología Comunicativa Crítica. Barcelona: El Roure Editorial. 
Greenwood, D.J. (2012). Doing and learning action research in the neo-liberal world of contemporary higher education. Action Research, 10(2), 115-132. DOI:

http://dx.doi.org/10.1177/1476750312443573

López, J. \& Ruiz, J.A. (2003). La ciudad de El Puerto de Santa María a través de la arqueología. El Puerto de Santa María (Cádiz): Concejalía de Cultura del Ayuntamiento de El Puerto de Santa María.

Malo, M. (2004). Prólogo. En M. Malo (Eds.), Nociones Comunes (pp. 13-40). Madrid: Traficantes de Sueños.

Manzano-Arrondo, V. (2012). La Universidad Comprometida. Vitoria: Hegoa.

Manzano-Arrondo, V. \& Suárez, E. (2015). Unidad de Acción Comprometida: una propuesta de solución ante el problema universitario del servicio a la sociedad. Hábitat y Sociedad, 8, 147-166.

Montero, M. (2009). Methods for liberation: Critical consciousness in action. En M. Montero \& C. Sonn (Eds.), Psychology of Liberation: Theory and Applications (pp. 73-92). Berlin: Springer.

Mori, M.P. (2008). Una propuesta metodológica para la intervención comunitaria. Liberabit, 14, 81-90.

Parrado, E.A., McQuiston, C. \& Flippen, C.A. (2005). Partiticating survey research. Integrating community collaboration and quantitative methods for the study of gender and HIV risks among hispanic migrants. Sociological Methods \& Research, 34(3), 204-239. DOI: http://dx.doi.org/10.1177/0049124105280202

Smith, L., Bratini, L., Chambers, D., Jensen, R.V. \& Romero, L. (2010). Between idealism and reality: Meeting the challenges of participatory action research. Action Research, 8(4), 407-425. DOI: http://dx.doi.org/10.1177/1476750310366043

Speck, B.W. (2001). Why Service-Learning?. New Directions for Higher Education, 114, 3-13. DOI: http://dx.doi.org/10.1002/he.8.abs

Strier, R. (2011). The construction of university-community partnerships: Entangled perspectives. Higher Education, 62, 81-97. DOI: http://dx.doi.org/10.1007/s10734-010-9367-x

Thiollent, M. (2011). Action research and participatory research: An overview. International Journal of Action Research, 7(2), 160-174.

Tracy, S.J. (2010). Qualitative quality: eight «Big-Tent» criteria for excellent qualitative research. Qualitative Inquiry, 16(10), 837-851. DOI: http://dx.doi.org/10.1177/1077800410383121

Van der Salm, C.A., Van Knippenberg, D. \& Daamen, D.D. (1997). A critical test of the choice questionnaire for collecting informed public opinion. Quality \& Quantity, 31, 193-917. DOI: http://dx.doi.org/10.1023/A:1004214500745

Whitley, J.R. (2002). A guide to organizing community forums. Boston: Community Catalyst. 